absence of intermixing between the lanthanum and cerium phosphates in nanoparticles. They noted that the core-shell structure of nanoparticles is also confirmed by high quantum yields of $80 \%$, while phosphors prepared by conventional solid-state methods have much lower quantum yields at grain sizes smaller than $0.5 \mu \mathrm{m}$.

Haase said, "Quantum yields so close to those obtained for macrocrystalline materials were, until recently, considered unattainable with nanocrystalline emitters."

The researchers believe that they will be able to further increase the quantum yield by adjusting the shell thickness and by using metal salts of very high purity.

EKATERINA A. LITVINOVA

\section{Proton Irradiation Induces \\ Magnetic Ordering in Graphite}

Macroscopic magnetic ordering phenomena found in highly oriented pyrolytic graphite (HOPG) are of great interest to the scientific community. A group of researchers from the University of Leipzig has focused on the fact that the saturation magnetization of carbon prepared from hydrogen-rich materials increases with an increase of hydrogen concentration. They note that the origin of magnetization might be related to a mixture of carbon atoms with $s p^{2}$ and $s p^{3}$ bonds. As reported in the November 28, 2003, issue of Physical Review Letters, P. Esquinazi and his colleagues used highly oriented pyrolytic graphite to show that implanted protons in HOPG triggers ferromagnetic (or ferrimagnetic) ordering with a Curie temperature above room temperature.

The researchers irradiated HOPG samples, containing a total content of magnetic metal impurities below 1 ppm, with a $2.25 \mathrm{MeV}$ proton microbeam. They demonstrated that, depending on the irradiation dose, the saturation magnetization of HOPG increases up to $5 \times 10^{-6}$ emu, almost a factor of 100 larger than that of the non-irradiated sample. The coercive field was found to be nearly the same for all irradiation doses ( 100 Oe) and was only weakly temperaturedependent. The researchers measured the maximum contribution of Fe impurities to the magnetic moment of the samples to be less than $6.1 \times 10^{-8} \mathrm{emu}$ and con- cluded that ferromagnetic ordering of HOPG is not correlated with magnetic metals. According to the temperaturedependent studies, the researchers indicated a Curie temperature in HOPG above $400 \mathrm{~K}$.

The researchers also performed magnetic force microscopy studies of the irradiated and non-irradiated areas. They showed the presence of homogeneously distributed magnetic domains with a periodicity of 2-4 $\mu \mathrm{m}$, depending on the irradiation dose. However, because of the small coercive field of the magnetic surface and the influence of the magnetic tip, the magnetic domain distribution depended on the distance between tip and surface. By measuring the phase shift as a function of tip-surface distance, the research team estimated the maximum magnetic moment observed by the tip to be $\sim 3 \times 10^{-15} \mathrm{~A} \mathrm{~m}^{2}$, and the total saturation magnetic moment to be $6 \times 10^{-6} \mathrm{emu}$.

The researchers concluded that their findings "open a new research field in magnetism with possible applications in spin electronics, since the electrons in the hydrogenated graphene should have a

\section{The Materials Gateway: www.mrs.org}

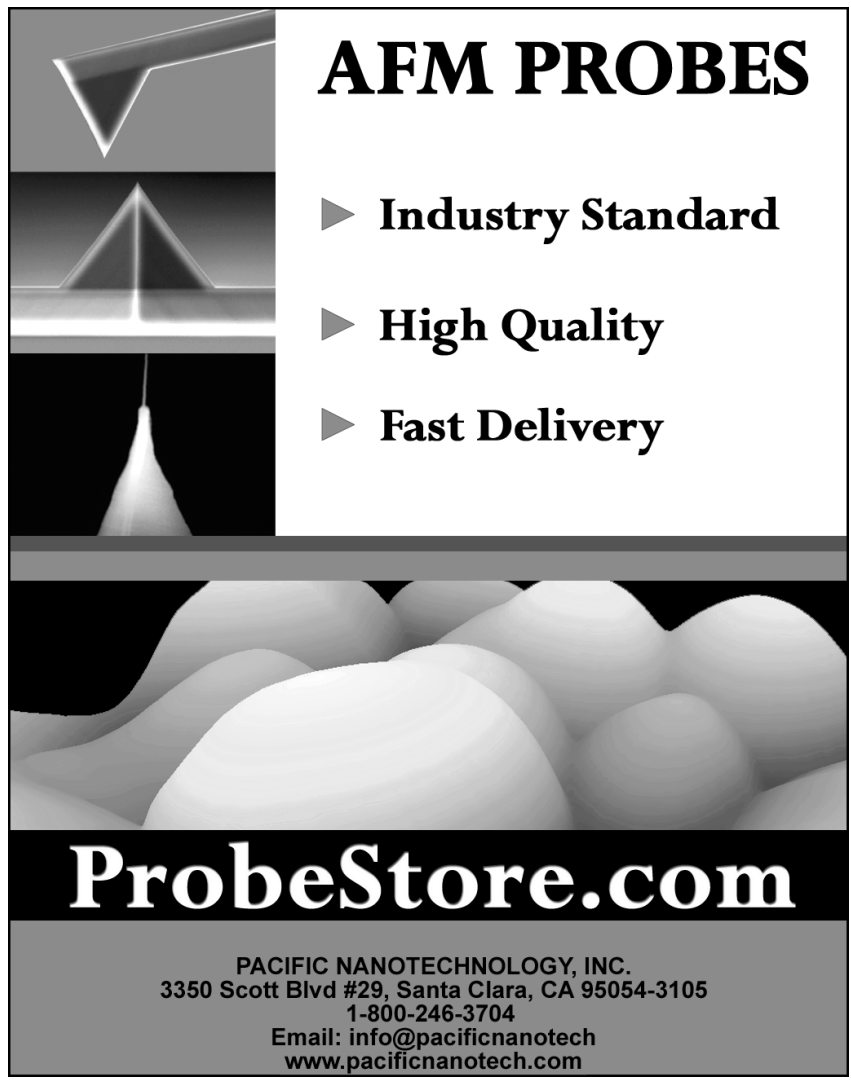

For more information, see http://advertisers.mrs.org

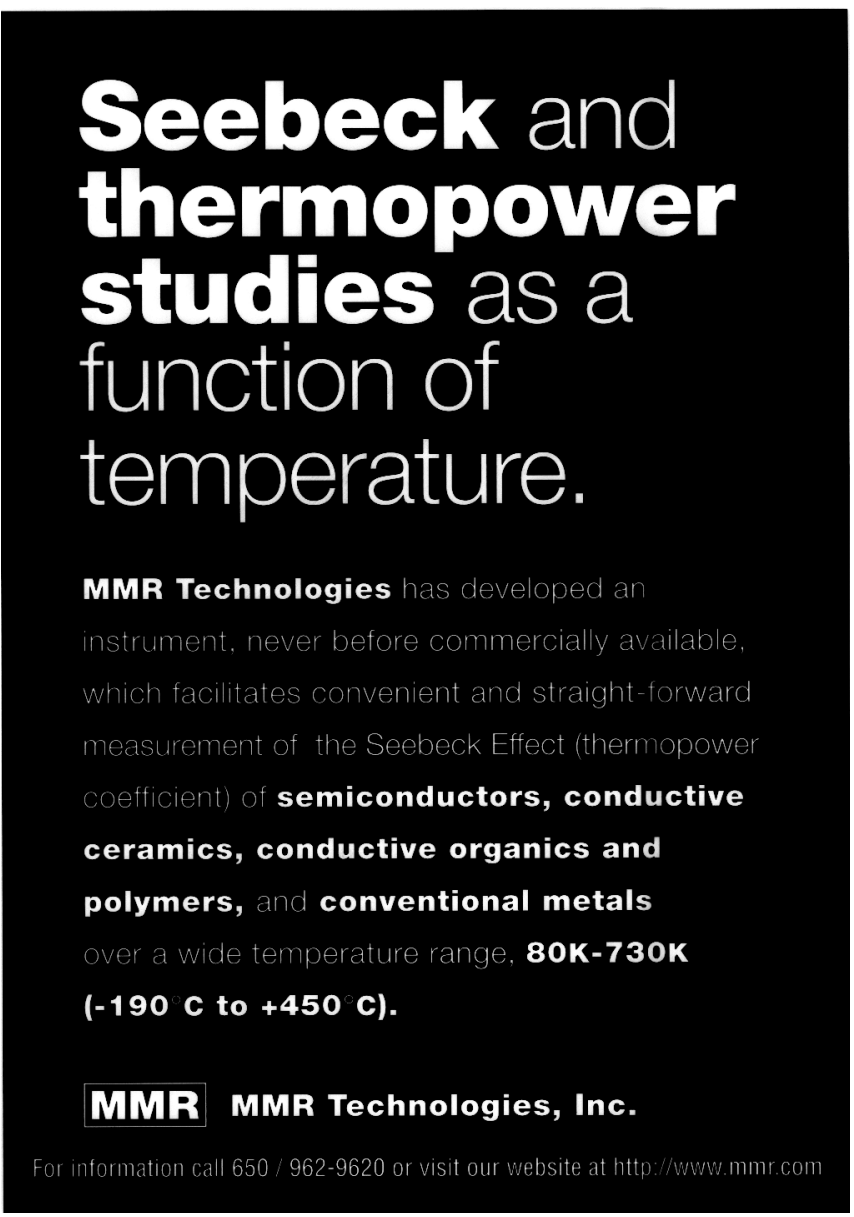

For more information, see http://advertisers.mrs.org 
very large spin polarization." In their related work published in Advanced Materials (15, 2003), the researchers showed that proton microbeam makes it possible to create magnetic spots on the graphite with diameters of only a few micrometers.

\section{ANDREI A. ELISEEV}

\section{CdS Nanoparticles on Si Self-Organize into Luminescent Nanorings}

Realization of nanometer-sized devices is dependent on the physical properties of nanostructures. A group of researchers from NTT, the Royal Institution of Great Britain, the University of Wales, and Yokohama National University has achieved a self-organization process of cadmium sulfide nanoparticles that results in the formation of luminescent nanometer-sized ring structures on a silicon wafer.

As reported in the September 16, 2003, issue of Advanced Materials, nanoparticles of CdS, $10 \mathrm{~nm}$ in size, were synthesized by the reaction between cadmium acetate and thioacetamide. This method enables control of the size and shape of the $\mathrm{CdS}$ nanoparticles. Planar silicon wafers of (111) orientation with a thin oxide layer $(<1 \mathrm{~nm})$ were used as the substrate. Nanoparticles were introduced onto the silicon wafer by dip coating from ethanol suspension, aided by an ultrasonic bath, at room temperature. On annealing of these $\mathrm{Si}$ substrates, in an ultrahigh-vacuum chamber at $800-850^{\circ} \mathrm{C}$, the $\mathrm{CdS}$ nanoparticles formed circular structures on the silicon surface. The nanorings, consisting of particles from $20 \mathrm{~nm}$ to $100 \mathrm{~nm}$, ranged in size from $100 \mathrm{~nm}$ to $1000 \mathrm{~nm}$. Information about particle size was obtained by atomic force microscopy. Mapping of tunneling electron luminescence from these nanoring structures showed a oneto-one correspondence between the topography and light emission. The resolution of this mapping technique enabled the observation of luminescence from individual nanoparticles (about 10-20 nm in size). The researchers said that the decomposition of the silicon oxide layer, followed by a drying phenomenon, leads to the formation of these luminescent nanorings.

The researchers said that "this material could be a potential candidate for developing optical components on silicon, if ultimate control of these structures can be achieved."

MAXIM NIKIFOROV

\section{InGaP/GaAs HBTs Operate as Light-Emitting Transistor}

The concept of wide-bandgap semiconductor emitters for minority carrier injection efficiency has been known since the work of Shockley in 1948 and Kroemer in 1953. This concept has been used to achieve high minority carrier injection efficiency in bipolar transistors, of which InP heterojunction bipolar transistors (HBTs) are the fastest, with operating speeds exceeding $450 \mathrm{GHz}$. In this approach, base-current recombination has been regarded as a source of undesired waste heat. However, as reported in the January 5 issue of Applied Physics Letters, M. Feng,
N. Holonyak Jr., and W. Hafez of the Department of Electrical and Computer Engineering at the University of Illinois at Urbana-Champaign have directly observed radiative recombination in the graded base layer of an $\mathrm{InGaP} / \mathrm{GaAs}$ HBT. The researchers were able to change the spontaneous light-emission intensity by varying the base current from $0 \mathrm{~mA}$ to $5 \mathrm{~mA}$. They demonstrated that the modulation of the light emission was in phase with the base current modulated in transistor operation at $1 \mathrm{MHz}$.

The scientists used metalorganic chemical vapor deposition to grow the InGaP/ GaAs HBT test structure consisting of a $3500 \AA n$-type GaAs collector, a $600 \AA$ carbon-doped and compositionally graded p-type InGaAs base with $1.4 \%$ In, an $800 \AA$ n-type InGaP emitter, and a $1000 \AA$-type InGaAs emitter contact cap. The tested HBT device consists of electron-beamdefined $\mathrm{Ti} / \mathrm{Pt} / \mathrm{Au}$ emitter contacts fabricated by using self-aligned emitter etch and base metal deposition. The researchers employed a bisbenzocyclobutene (BCB) based etch-back process for back-end fabrication to render the electrode and contact formation on the top of the transistor.

The researchers observed no light emission for zero base current, while at $1 \mathrm{~mA}$, they observed the first weak light emission that increased in strength when the base current was increased to $5 \mathrm{~mA}$. The output emission wavelength of $885 \mathrm{~nm}$ is consistent with the energy bandgap of the compositionally graded InGaAs base of the HBT. The researchers also performed tests of output light modulation at $1 \mathrm{MHz}$

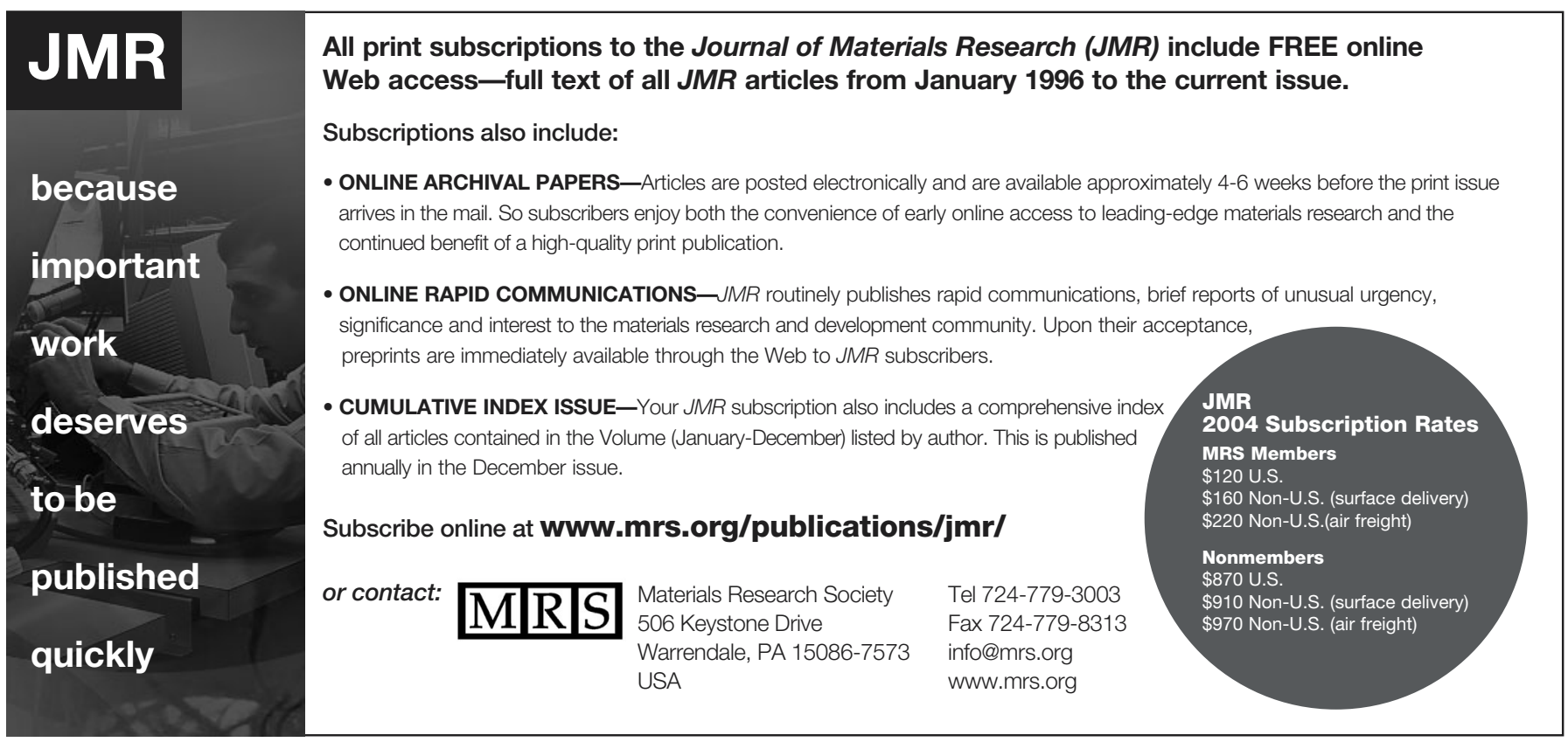

